\title{
Generador lineal para un generador eólico de baja potencia, selección, diseño y simulación en comsol multiphysic
}

\section{Linear generator for a low power wind generator, selection, design and simulation in comsol mulliphysic}

\author{
José German Mora Santos ${ }^{1}$ (D), Edwin Rivas Trujillo² (iD), Henry Montaña Quintero
}

Para citar este articulo: J. G. Mora Santos, E. Rivas Trujillo, H. Montaña Quintero, "Generador lineal para un generador eólico de baja potencia, selección, diseño y simulación en comsol multiphysic". Revista Vínculos, vol. 17, no. 2, pp. 120-128, julio-diciembre, 2020. https://doi.org/10.14483/2322939X.18620

Recibido: 30-10-2020 / Aprobado: 20-12-2020

\section{Resumen}

En el presente documento se expone un estudio comparativo de tres topologías propuestas de generador lineal, a partir de la recopilación de los estudios consignados en la literatura científica, son modeladas por medio de simulaciones en 2D en COMSOL y se analizan los resultados obtenidos tomando como indicador de desempeño la potencia de salida y el comportamiento bajo carga, como contribución al proyecto de investigación TOPOLOGÍAS DE GENERADOR EÓLICO DE BAJA POTENCIA. Los resultados obtenidos son satisfactorios resaltando la primera de las topologías propuestas, que presenta el mejor comportamiento bajo carga y un valor adecuado de potencia de salida para aplicaciones de baja potencia. Gracias al empleo de herramientas computacionales se evita la construcción de varios prototipos, lo que da la posibilidad de generar un banco virtual de pruebas para futuros desarrollos en esta área y lograr un avance en la investigación de estos dispositivos en Colombia.

Palabras clave: Electromagnetismo, Elementos Finitos, Flujo Magnético, Generador Lineal, Imanes Permanentes, Simulación.

\begin{abstract}
This document presents a comparative study of three proposed linear generator topologies, based on the compilation of studies reported in the scientific literature, they are modeled by means of 2D simulations in COMSOL and the results obtained are analyzed taking output power and behavior under load as a performance indicator, as a contribution to the research project TOPOLOGIES OF LOW POWER WIND GENERATOR. The results obtained are satisfactory, highlighting the first of the proposed topologies, which presents the best behavior under load and an adequate output power value for low power applications. Thanks to the use of computational tools, the construction of several prototypes is avoided, which gives the possibility of generating a virtual test bench for future developments in this area and achieving a breakthrough in the research of these devices in Colombia.
\end{abstract}

Keywords: Electromagnetism, Finite elements, Linear generator, Magnetic flux, Permanent Magnets, Simulation.

\footnotetext{
Engineering Electrical Student: Universidad Distrital Francisco José de Caldas, Colombia. E-mail: jgmoras@correo.udistrital.edu.co Tenured Professor: Universidad Distrital Francisco José de Caldas, Colombia. E-mail: erivas@udistrital.edu.co

Engineering Doctorate Student: Universidad Distrital Francisco José de Caldas, Colombia. E-mail: hmontanaq@correo.udistrital.edu.co
} 


\section{Introducción}

Las máquinas eléctricas de inducción son dispositivos capaces de convertir energía mecánica en energía eléctrica cuando su funcionamiento es el de un generador, o viceversa si funcionan como motor, basándose en la ley de inducción de Faraday al utilizar el campo electromagnético como soporte para dicha conversión [1].

Estas máquinas aprovechan el movimiento rotatorio o lineal, existiendo una serie de características que diferencian a las máquinas lineales de las máquinas rotativas, y que las hacen muy interesantes en determinadas aplicaciones y contextos, como, por ejemplo, un mejor comportamiento a bajas velocidades y el no necesitar acoples mecánicos intermedios para la conversión de energía [2, 3].

Cualquier tipo de máquina rotativa se puede adaptar y construir desde una perspectiva lineal, ver figura 1, por lo que han sido empleadas ampliamente en la industria debido a su estructura simple y de bajo costo de construcción [2, 4, 5-7].

Figura 1. Adaptación de una máquina rotativa a lineal.

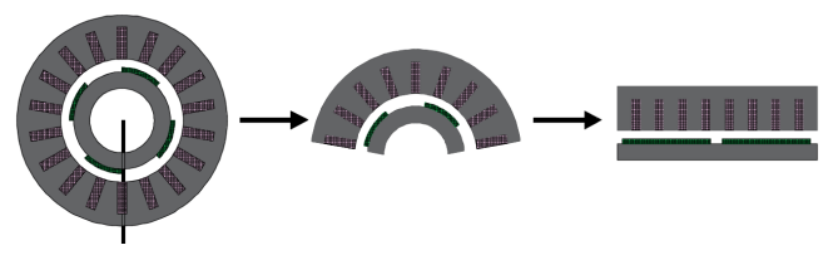

Fuente: elaboración propia.

Las aplicaciones más conocidas de los generadores lineales están relacionadas con el área del transporte, dado que en el proceso de inducción estos dispositivos además de entregar propulsión pueden generar levitación, lo que los hace comunes en aplicaciones de transporte de trenes de alta velocidad, ya que además ofrecen una ventaja adicional al no existir rozamiento durante su operación $[2,5,8,9]$.

Gracias a que los generadores lineales ofrecen la posibilidad de convertir directamente energía mecánica en energía eléctrica, sin pasos intermedios entre la interfaz primaria y la máquina eléctrica, es común que también sean usados en el campo de la energía undimotriz acoplados a boyas [10-14].

Por su estructura (figura 2), convierten la energía por medio de un movimiento oscilante sobre un solo eje coordenado de manera periódica, a velocidad reducida. El voltaje generado, la energía eléctrica y, por lo tanto, la corriente de armadura es directamente proporcionales a la velocidad del traslator [15-17].

Figura 2. Estructura de un generador lineal de imanes permanentes.

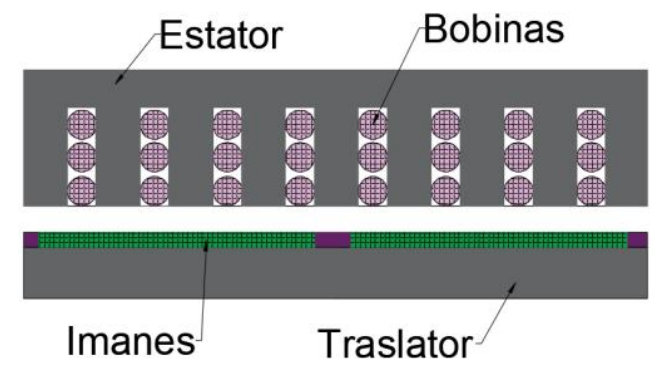

Fuente: elaboración propia.

El análisis de este tipo de generadores puede realizarse partiendo del realizado a los generadores rotativos, si se tiene en cuenta dos aspectos: que las dimensiones y desplazamientos angulares deben reemplazarse por dimensiones y desplazamientos lineales; y que se debe remplazar los pares por fuerzas. La parametrización del generador lineal como la estructura de los resultados se obtienen de manera similar [5, 18]. En la Tabla 1 se presenta las ventajas y desventajas comparativas de generadores lineales y rotativos.

Tabla 1. Ventajas y desventajas comparativas de generadores lineales y rotativos $[2,3,5,15,19-21]$.

\begin{tabular}{|c|c|}
\hline Ventajas & Desventajas \\
\hline $\begin{array}{l}\text {-1 } \text { Alta eficiencia } \\
\text { - } \text { Menor tamaño y peso } \\
\text { - Sistema mecánico más ligero y simple } \\
\text { - } \text { Alta densidad de potencia } \\
\text { - Mayor velocidad } \\
\text { - } \text { Mayor aceleración cuando es tiempo de mecanizado } \\
\text { - } \text { Niveles bajos de ruido } \\
\text { - Reducción de vibraciones } \\
\end{array}$ & $\begin{array}{l}\text { - Necesidad de disipar calor por medio } \\
\text { de sistemas de refrigeración } \\
\text { Reducción de los niveles de precisión, } \\
\text { cuando no hay un incremento } \\
\text { controlado de la temperatura } \\
\text { - Aumento del costo de las soluciones } \\
\text { cuando su diseño es complejo } \\
\text { - No posee elementos de trasmisión para } \\
\text { amortiguar cualquier perturbación } \\
\text { mecánica }\end{array}$ \\
\hline $\begin{array}{l}\text { Menores pérdidas por fricción, lo que } \\
\text { conlleva a una disminución en los } \\
\text { costos del mantenimiento }\end{array}$ & $\begin{array}{l}\text { Para cambios de carga es necesario } \\
\text { utilizar un controlador electrónico } \\
\text { extremadamente rápido para mantener } \\
\text { la estabilidad } \\
\text { - Uso habitual de técnicas de filtrado } \\
\text { para mitigar resonancia mecánica en } \\
\text { condiciones exigentes }\end{array}$ \\
\hline
\end{tabular}

Fuente: elaboración propia. 
Hasta el momento se han desarrollado diferentes configuraciones de generadores lineales de imanes permanentes, cada topología empleada o característica constructiva modificada, está pensada en mejorar ciertos aspectos de la máquina y/o solucionar problemas que se presentan en esta, por lo que es posible clasificarlas como se propone en la tabla 2.

Tabla 2. Clasificación de las topologías de acuerdo a su enfoque.

\begin{tabular}{|c|c|c|c|}
\hline Clasificación & Subdivisión & \begin{tabular}{|c|}
$\begin{array}{c}\text { Aspecto que } \\
\text { mejora }\end{array}$ \\
\end{tabular} & $\begin{array}{c}\text { Aspecto a tener en } \\
\text { cuenta }\end{array}$ \\
\hline $\begin{array}{l}\text { Pensadas en la } \\
\text { geometría }\end{array}$ & $\begin{array}{ll}\text { - } & \text { Plano } \\
\text { - } & \text { Tubular } \\
\text { Multilateral } \\
\end{array}$ & $\begin{array}{l}\text { Flujos de } \\
\text { dispersión y } \\
\text { eficiencia }\end{array}$ & $\begin{array}{l}\text { Tiempo y costos de } \\
\text { construcción }\end{array}$ \\
\hline $\begin{array}{l}\text { Pensadas en la ubicación } \\
\text { de imanes y bobinas }\end{array}$ & $\begin{array}{l}\text { - De bobina móvil } \\
\text { - De imanes móviles } \\
\end{array}$ & $\begin{array}{l}\text { Tiempo de } \\
\text { respuesta y } \\
\text { eficiencia. }\end{array}$ & $\begin{array}{l}\text { Refrigeración } \\
\text { efectiva y masa en } \\
\text { movimiento. }\end{array}$ \\
\hline $\begin{array}{l}\text { Pensadas en el } \\
\text { entrehierro }\end{array}$ & $\begin{array}{l}\text { - De imanes grandes } \\
\text { De imanes } \\
\text { pequeños }\end{array}$ & $\begin{array}{l}\text { Densidad de flujo } \\
\text { magnético en el } \\
\text { entrehierro }\end{array}$ & $\begin{array}{l}\text { Fuerza de atracción } \\
\text { entre imanes }\end{array}$ \\
\hline $\begin{array}{c}\text { Pensadas en el efecto } \\
\text { cogging }\end{array}$ & $\begin{array}{l}\text { De armadura } \\
\text { ranurada } \\
\text { De armadura sin } \\
\text { ranurar } \\
\end{array}$ & $\begin{array}{l}\text { Armónicos, par } \\
\text { frenado, eficiencia } \\
\text { y efecto cogging }\end{array}$ & $\begin{array}{l}\text { Cantidad de densidad } \\
\text { de flujo }\end{array}$ \\
\hline $\begin{array}{l}\text { Pensadas en la relación } \\
\text { Estator-Traslator }\end{array}$ & $\begin{array}{ll}\text { - Estator corto } \\
\text { movible } \\
\text { - Estator corto fijo } \\
\text { - } \text { Traslator corto } \\
\text { movible } \\
\text { - Bilateral con } \\
\text { traslator externo } \\
\text { - Bilateral con } \\
\text { traslator interno }\end{array}$ & $\begin{array}{l}\text { Superficie activa } \\
\text { de la interacción } \\
\text { estator-traslator y } \\
\text { densidad de } \\
\text { potencia }\end{array}$ & \begin{tabular}{|l} 
Costo de \\
construcción, \\
pérdidas Joule y \\
Efecto fin
\end{tabular} \\
\hline $\begin{array}{c}\text { Pensadas en la } \\
\text { disposición de los imanes }\end{array}$ & $\begin{array}{ll}\text { - } & \text { De disposición } \\
\text { apilada } \\
\text { De disposición } \\
\text { superficial }\end{array}$ & $\begin{array}{l}\text { Eficiencia, } \\
\text { potencia y ángulo } \\
\text { de carga de la } \\
\text { máquina }\end{array}$ & $\begin{array}{l}\text { Masa total de los } \\
\text { imanes, transitorios y } \\
\text { cortocircuitos }\end{array}$ \\
\hline $\begin{array}{l}\text { Pensadas en los } \\
\text { patrones de } \\
\text { magnetización }\end{array}$ & $\begin{array}{ll} & \text { Radial } \\
& \text { Axial } \\
\text { - } & \text { Halbach } \\
& \text { Cuasi-Halbach } \\
\end{array}$ & $\begin{array}{l}\text { Densidad de flujo, } \\
\text { distorsión } \\
\text { armónica y } \\
\text { potencia de salida } \\
\end{array}$ & $\begin{array}{l}\text { Distancia entre los } \\
\text { puntos finales de } \\
\text { cada imán (flujos de } \\
\text { fuga) }\end{array}$ \\
\hline \multirow[t]{2}{*}{$\begin{array}{c}\text { Pensadas en la } \\
\text { concatenación del flujo } \\
\text { magnético }\end{array}$} & $\begin{array}{l}\text { - De flujo longitudinal } \\
\text { - De flujo transversal } \\
\text { De núcleo de aire }\end{array}$ & \begin{tabular}{|lr} 
Flujos & de \\
dispersión & $y$ \\
potencia & nominal
\end{tabular} & $\begin{array}{l}\text { Construcción simple, } \\
\text { magnitud de la } \\
\text { reactancia }\end{array}$ \\
\hline & & $\begin{array}{l}\text { por área de } \\
\text { espacio de aire }\end{array}$ & $\begin{array}{l}\text { sincrónica, } \\
\text { fluctuaciones de } \\
\text { empuje y necesidad } \\
\text { de compensación }\end{array}$ \\
\hline
\end{tabular}

Fuente: elaboración propia.

Con el objeto de proponer las topologías a comparar es necesario considerar algunos aspectos que aseguren que el diseño del generador otorgue resultados satisfactorios, reuniendo ciertas características que se adecuen a las posibles aplicaciones del generador lineal en bajas potencias [22].

Las dimensiones óptimas para el generador se obtienen del análisis de la densidad de corriente máxima permitida y el número mínimo permitido de polos, teniendo en cuenta las combinaciones más comunes de ranuras y polos que se exponen en la tabla 3, por lo tanto, estas dimensiones se pueden obtener minimizando el imán o la masa de cobre [2, 3]. El diseño de cada una de las topologías tiene en cuenta las ecuaciones consignadas en la tabla 4 obtenidas de la teoría electromagnética.

Tabla 3. Combinaciones de polos magnéticos y ranuras [23].

\begin{tabular}{|l|c|c|c|c|c|c|}
\hline Ranuras & 3 & 6 & 12 & 21 & 36 & 48 \\
\hline Polos & 2 & 2 & 2 & 2 & 2 & 2 \\
\cline { 2 - 7 } & 4 & 4 & 4 & 4 & 4 & 4 \\
\cline { 2 - 7 } & & 8 & 8 & 8 & 6 & 8 \\
\cline { 2 - 7 } & & 10 & 10 & 14 & 8 & 10 \\
\cline { 2 - 7 } & & & 16 & 16 & 10 & 14 \\
\cline { 2 - 7 } & & & $\ldots$ & $\ldots$ & $\ldots$ & $\ldots$ \\
\hline
\end{tabular}

Fuente: elaboración propia.

Tabla 4. Fórmulas de diseño.

\begin{tabular}{|c|c|c|}
\hline Variable & Fórmula & Donde \\
\hline $\begin{array}{l}\text { Área transversal mínima el } \\
\text { conductor }\end{array}$ & $A_{C, \text { minimum }}=\frac{I}{J c}$ & $\begin{array}{c}\text { I = Corriente máxima } \\
\text { permitida } \\
\text { Jc }=\text { Densidad de corriente }\end{array}$ \\
\hline $\begin{array}{l}\text { Número de vueltas por } \\
\text { bobina }\end{array}$ & $\mathrm{N}_{\text {coll }}=\frac{\mathrm{F}_{\mathrm{Il}} * \mathrm{~h} * \mathrm{~W}}{\mathrm{Ac}}$ & $\begin{array}{c}\mathrm{F}_{\mathrm{Ul}}=\text { Factor de llenado } \\
\mathrm{h}=\text { Altura de la ranura } \\
\mathrm{W}=\text { Ancho de la ranura } \\
\mathrm{Ac}=\text { Área transversal del } \\
\text { conductor seleccionado }\end{array}$ \\
\hline Número de vueltas por fase & $\begin{array}{l}\mathrm{N}_{\text {phase }}=\mathrm{C}_{\text {phase }} * \mathrm{~N}_{\text {coil }} \\
\mathrm{N}_{\text {phase }}=\mathrm{q} * \text { pu } * \mathrm{~N}_{\text {coil }}\end{array}$ & $\begin{array}{c}\mathrm{N}_{\text {coill }}=\text { Número de vueltas por } \\
\text { bobina } \\
\mathrm{C}_{\text {phase }}=\text { Bobinas por fase } \\
\mathrm{Pu}=\text { pares de polos útiles }\end{array}$ \\
\hline & & $q=$ Ranuras por polo y fase \\
\hline Carga lineal & $\mathrm{L}_{\text {linear }}=\frac{\mathrm{N}_{\mathrm{coll}} * \mathrm{I}}{\tau}$ & $\begin{array}{c}\mathrm{N}_{\text {coil }}=\begin{array}{c}\text { Número de vueltas por } \\
\text { bobina }\end{array} \\
\mathrm{I}=\text { corriente máxima } \\
\text { permitida } \\
\tau=\text { paso de ranura }\end{array}$ \\
\hline Longitud del conductor & $L_{c}=(2 * W+2 * P) * N_{\text {phase }}$ & $\begin{array}{c}\text { W }=\text { Ancho de la ranura } \\
\mathrm{P}=\text { Profundidad de la ranura } \\
\mathrm{N}_{\text {phase }}=\text { Número de vueltas } \\
\text { por fase }\end{array}$ \\
\hline Resistencia por fase & $\mathrm{R}_{\text {phase }}=\mathrm{L}_{\mathrm{c}} * \mathrm{R}_{\mathrm{c}}$ & $\begin{array}{c}\mathrm{L}_{\mathrm{c}}=\text { Longitud del conductor } \\
\mathrm{R}_{\mathrm{c}}=\begin{array}{l}\text { Resistencia del } \\
\text { conductor }\end{array}\end{array}$ \\
\hline FEM generada & $\begin{aligned} \mathrm{FEM}=2 * \mathrm{k}_{\mathrm{ff}} * & \mathrm{k}_{\mathrm{d}} * \mathrm{~N}_{\text {phase }} \\
& * \mathrm{~V}_{\text {maximum }} \\
& * \mathrm{~L}_{\text {stator }} * \mathrm{~B}\end{aligned}$ & $\begin{array}{c}\mathrm{k}_{\mathrm{ff}}=\text { Factor de forma } \\
\mathrm{k}_{\mathrm{d}}=\text { Factor de distribución } \\
\mathrm{V}_{\text {maximum }}=\text { Velocidad máxima } \\
\mathrm{L}_{\mathrm{stator}}=\text { Longitud del estator } \\
\mathrm{B}=\text { Densidad de flujo } \\
\text { magnético en el entrehierro }\end{array}$ \\
\hline $\begin{array}{l}\text { Resistencia de carga de } \\
\text { diseño }\end{array}$ & $R_{10 w}=\frac{(F E M)^{2}}{P_{\text {design }}}-R_{\text {phase }}$ & $\begin{aligned} & \text { FEM }=\text { Fuerza electromotriz } \text { generada } \\
&= \\
& \mathrm{P}_{\text {design }}=\text { Potencia de diseño } \\
& \mathrm{R}_{\text {phase }}=\text { Resistencia por fase }\end{aligned}$ \\
\hline
\end{tabular}

Fuente: elaboración propia.

Para el material de los imanes se realiza una comparativa entre imanes de neodimio e imanes de ferrita con base en la literatura científica, encontrando mejores características y resultados con imanes de neodimio, como resultado se determina las principales ventajas y desventajas de estos imanes como se muestra en la tabla 5. 
Tabla 5. Ventajas y desventajas de los imanes de neodimio $[6,24]$.

\begin{tabular}{|c|c|}
\hline Ventajas & $\begin{array}{c}\text { Desventajas } \\
\end{array}$ \\
\hline $\begin{array}{l}\text { - Capacidad de permitir nuevos diseños } \\
\text { - } \\
\text { remanencia } \\
\text { - Propiedades por encima de las } \\
\text { tradicionales } \\
\text { - Menor masa con respecto a los imanes } \\
\text { de ferrita }\end{array}$ & $\begin{array}{l}\text { - Su utilización está condicionada por la } \\
\text { temperatura } \\
\text { - Sensibilidad al calor, no puede trabajar a } \\
\text { temperaturas por encima de los } 80^{\circ} \mathrm{C} \\
\text { - Manipulación cuidadosa dada su } \\
\text { fragilidad }\end{array}$ \\
\hline
\end{tabular}

Fuente: elaboración propia.

Una vez diseñadas las topologías, para lograr una comparativa entre ellas se emplea el método de elementos finitos, dado que, por lo general, este análisis es uno de los enfoques más poderosos para diseño y modelado precisos de máquinas eléctricas [2].

La principal ventaja del método es su capacidad para tratar cualquier tipo de geometría y material no homogéneo sin necesidad de alterar la formulación o el código de ordenador. Es decir, se proporciona una fidelidad geométrica y un tratamiento al material sin restricciones. Esto hace que sea uno de los métodos numéricos más utilizado en todas las ramas de la ingeniería $[6,22]$.

\section{Topologías propuestas}

Para la primera de las topologías, se propone un generador plano monolateral de núcleo ranurado de hierro con imanes móviles, y una concatenación del flujo magnético de manera longitudinal para obtener una reactancia sincrónica pequeña. Adicionalmente, se determina una disposición apilada de imanes pequeños para evitar cortocircuitos y esfuerzos mecánicos grandes, y se utiliza un patrón de magnetización Halbach para el traslator de mayor longitud.

Con base en el estudio realizado en [25] en donde se afirma que la baja densidad de flujo implica que se necesitan más vueltas para obtener un voltaje específico, se proponen 4 bobinas por fase en el estator para obtener una mejor distribución de vueltas por fase, y se adopta un devanado de tres capas para reducir el material del devanado y por consiguiente las pérdidas en el cobre, la configuración resultante para cuatro bobinas por fase se muestra en la figura 3.
Figura 3. Estator con devanado de tres capas con número par de polos.

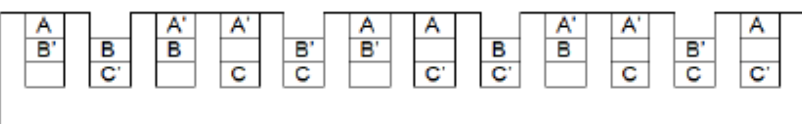

Fuente: elaboración propia.

La potencia esperada se define en $10 \mathrm{~W}$ por fase y la resistencia de carga se estima en 462 ohms para una fem generada de $68.09 \mathrm{~V}$ a una velocidad de $1 \mathrm{~m} / \mathrm{s}$ y un entrehierro de $5 \mathrm{~mm}$, en la figura 4 se observa la estructura resultante.

Figura 4. Primera topología propuesta.

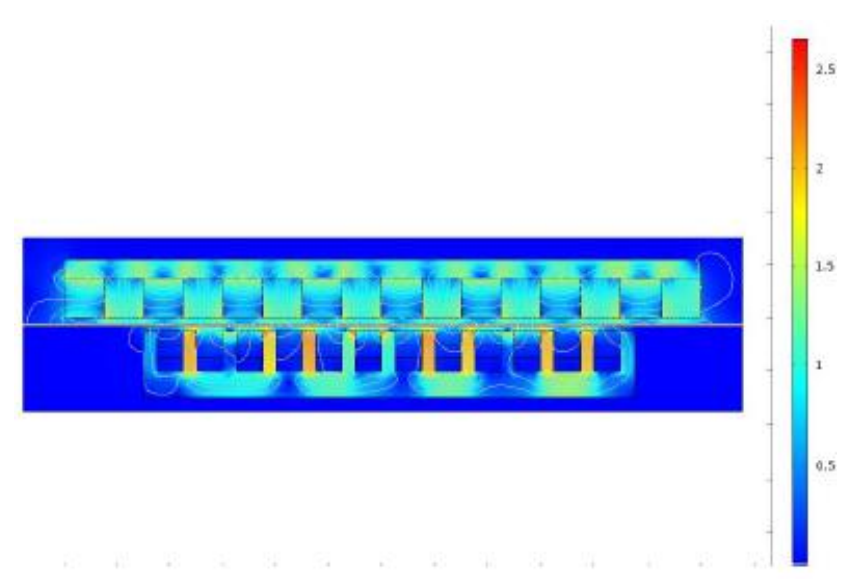

Fuente: elaboración propia.

Para la segunda topología a proponer, se piensa en ahorrar el espacio ocupado por el generador sin comprometer las características de la primera topología propuesta, para esto, se realiza las siguientes modificaciones: se escoge un tamaño de imanes grande para reducir el número de polos del generador y por ende la longitud del mismo, se emplea un patrón de magnetización axial y se determina un generador bilateral.

Hay que tener en cuenta que un generador de doble cara produce más potencia a costa de un pequeño aumento de cogging, lo que permitirá reducir el espacio ocupado por el generador para producir la misma potencia que en la topología uno [26]. 
Para reducir el aumento de cogging en el generador, en [27] descubrieron que esta fluctuación de empuje puede reducirse cambiando la forma del diente en ambos extremos del estator, por lo tanto, se adopta una estructura de dientes de extremo corto para que el estator reduzca la fluctuación de empuje y no comprometa las características técnicas del dispositivo como se observa en la figura 5 .

Figura 5. Estator bilateral con devanado de tres capas.

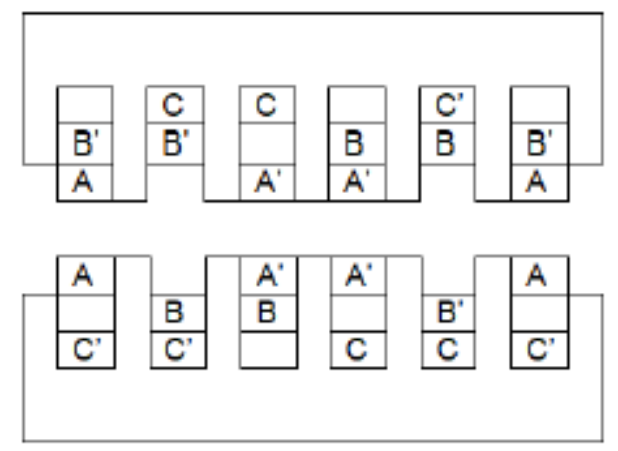

Fuente: elaboración propia.

La potencia esperada se define en $10 \mathrm{~W}$ por fase y la resistencia de carga se estima en 32 ohms para una fem generada de $17.84 \mathrm{~V}$ por estator a una velocidad de 1 $\mathrm{m} / \mathrm{s}$ y un entrehierro de $5 \mathrm{~mm}$, en la figura 6 se observa la estructura resultante.

Figura 6. Segunda topología propuesta.
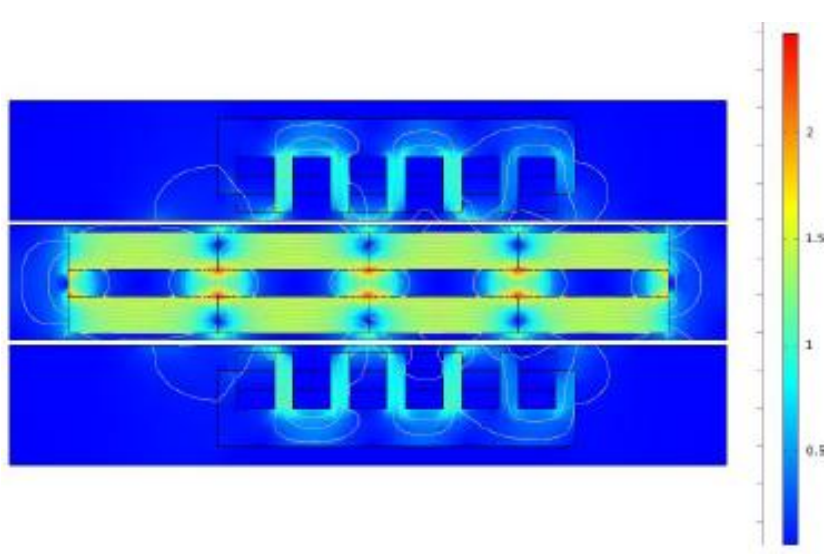

Para la tercera topología a proponer, el interés está centrado en disminuir el material ferromagnético y el alambre de cobre para los devanados en comparación con las demás topologías propuestas, para lo cual, se adopta un estator interno para cada fase con un devanado independiente de una capa, y se escoge dos tamaños de imanes para un patrón de magnetización Halbach en los traslator externos. Dado que el estator se dividirá en tres partes con devanados independientes, la configuración resultante de estos de una capa para dos bobinas por fase se muestra en la figura 7 .

Figura 7. Estator con devanado independiente.

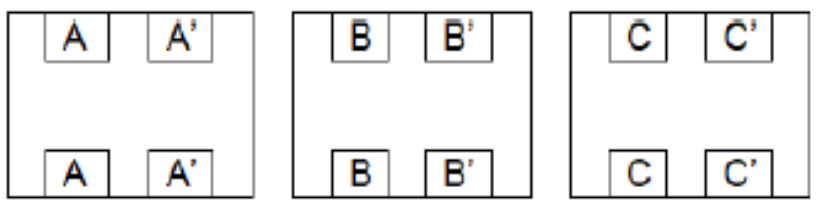

Fuente: elaboración propia.

La potencia esperada se define en $10 \mathrm{~W}$ por fase y la resistencia de carga se estima en 178 ohms para una fem generada de $42.33 \mathrm{~V}$ por estator a una velocidad de $1 \mathrm{~m} / \mathrm{s}$ y un entrehierro de $5 \mathrm{~mm}$, en la figura 8 se observa la estructura resultante.

Figura 8. Tercera topología propuesta.

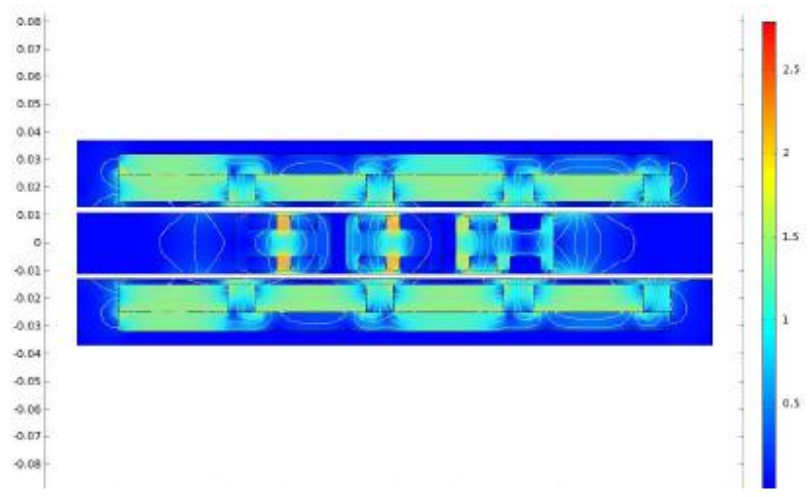

Fuente: elaboración propia.

Fuente: elaboración propia.
Las dimensiones de las tres topologías propuestas se exponen en la tabla 6. 
Tabla 6. Dimensiones de las topologías propuestas.

\begin{tabular}{|c|c|c|c|}
\hline Topología & Variable & Cantidad & Dimensiones \\
\hline \multirow{9}{*}{ Primera } & Imanes & 16 & $15 \times 15 \times 40 \mathrm{~mm}$ \\
\hline & Ranuras totales & 12 & $10 \times 15 \times 40 \mathrm{~mm}$ \\
\hline & Dientes totales & 13 & $5 \times 15 \times 40 \mathrm{~mm}$ \\
\hline & Estatores & 1 & $185 \times 25 \times 40 \mathrm{~mm}$ \\
\hline & Traslators & 1 & $240 \times 22 \times 40 \mathrm{~mm}$ \\
\hline & Vueltas totales Bobinado & 368 & $22 \mathrm{AWG}$ \\
\hline & Resistencia de carga & $462 \Omega$ & $\ldots$ \\
\hline & Voltaje inducido esperado & $68.09 \mathrm{~V}$ & $\ldots$ \\
\hline & Potencia por fase esperada & $10 \mathrm{~W}$ & $\ldots$ \\
\hline \multirow{9}{*}{ Segunda } & Imanes & 8 & $40 \times 10 \times 40 \mathrm{~mm}$ \\
\hline & Ranuras totales & 12 & $10 \times 15 \times 40 \mathrm{~mm}$ \\
\hline & Dientes totales & $\begin{array}{l}10 \text { internos } \\
4 \text { extremos }\end{array}$ & $\begin{array}{l}5 \times 15 \times 40 \mathrm{~mm} \\
5 \times 10 \times 40 \mathrm{~mm}\end{array}$ \\
\hline & Estatores & 2 & $185 \times 25 \times 40 \mathrm{~mm}$ \\
\hline & Traslators & 1 & $160 \times 27 \times 40 \mathrm{~mm}$ \\
\hline & Vueltas totales Bobinado & 184 & $22 \mathrm{AWG}$ \\
\hline & Resistencia de carga & $32 \Omega$ & $\ldots$ \\
\hline & Voltaje inducido esperado & $17.84 \mathrm{~V}$ & - \\
\hline & Potencia por fase esperada & $10 \mathrm{~W}$ & -- \\
\hline \multirow{9}{*}{ Tercera } & Imanes & 16 & $\begin{array}{l}40 \times 10 \times 40 \mathrm{~mm} \\
10 \times 10 \times 40 \mathrm{~mm}\end{array}$ \\
\hline & Ranuras totales & 12 & $10 \times 5 \times 40 \mathrm{~mm}$ \\
\hline & Dientes totales & 18 & $5 \times 5 \times 40 \mathrm{~mm}$ \\
\hline & Estatores & 3 & $35 \times 20 \times 40 \mathrm{~mm}$ \\
\hline & Traslators & 2 & $200 \times 17 \times 40 \mathrm{~mm}$ \\
\hline & Vueltas totales Bobinado & 92 & $22 \mathrm{AWG}$ \\
\hline & Resistencia de carga & $178 \Omega$ & - \\
\hline & Voltaje inducido esperado & $42.33 \mathrm{~V}$ & - \\
\hline & Potencia por fase esperada & $10 \mathrm{~W}$ & $\ldots$ \\
\hline
\end{tabular}

Fuente: elaboración propia.

\section{Resultados}

Para la obtención de resultados muy cercanos a la realidad y evitar la construcción de prototipos que generen costos adicionales de fabricación, se emplea software computacional capaz de resolver problemas no analíticos a través del uso del método de elementos finitos.

Tabla 7. Resultados del modelado con elementos finitos.

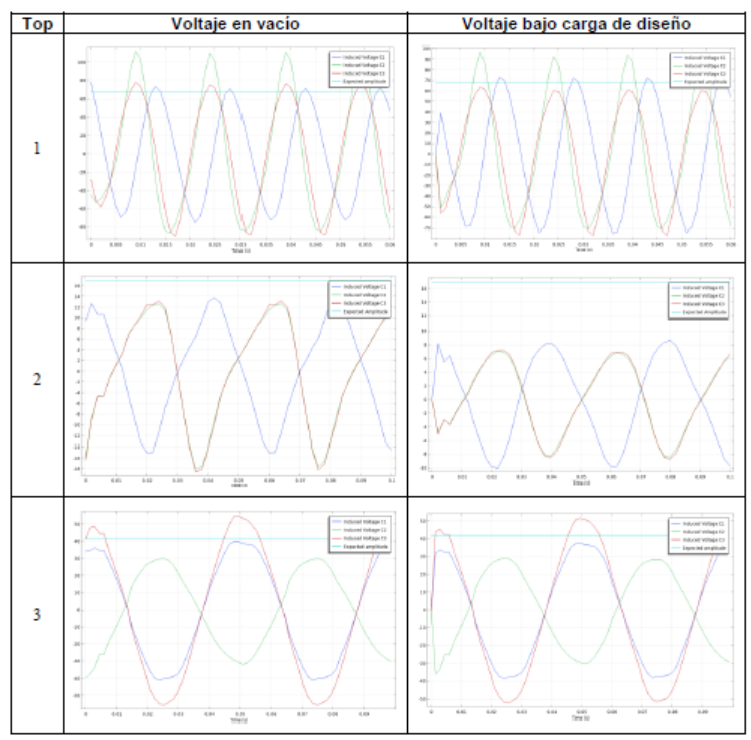

Fuente: elaboración propia.
El modelado de cada una de las topologías fue realizado para que los generadores estuviesen en vacío y posteriormente bajo la carga de diseño, obteniéndose los resultados que se muestran en la tabla 7 . Las magnitudes esperadas se grafican como constantes.

Con el fin de comparar el indicador de desempeño de potencia de cada topología bajo la misma carga, se realizan simulaciones conectando a cada generador la carga de diseño de las otras dos topologías adicionales a la propia, y se consignan los promedios de potencia por fase del generador en la figura 9. Se observa que la salida de potencia para cualquier carga es mayor en la topología uno, siendo esta la de mejor desempeño.

Figura 9. Variación de la potencia a diferentes cargas.

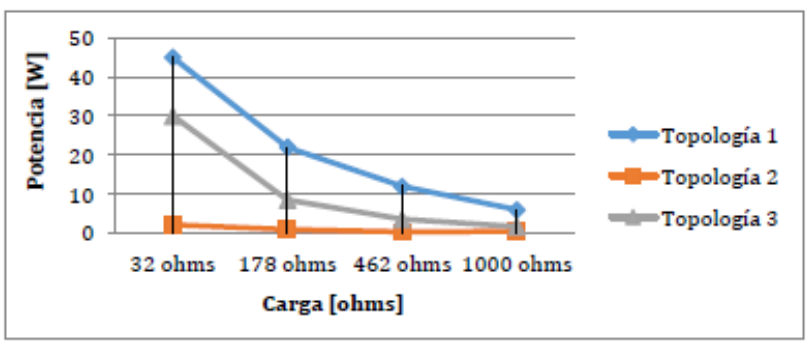

Fuente: elaboración propia.

\section{Conclusiones}

A pesar de que la segunda topología reduce el espacio ocupado por el generador, presenta perturbaciones de onda y una reducción de la tensión en bornes bajo carga, lo que conlleva a presentar la menor salida de potencia de todas las topologías propuestas, siendo la topología uno la de mejor comportamiento y salida de potencia en condiciones de carga.

Como resultado de la modificación de los dientes extremos en la topología dos se observa una disminución de la densidad de flujo magnético en los dientes extremos, lo que reduce proporcionalmente la fuerza dentada o efecto cogging en el generador, sin embargo, se presentan pequeños flujos de dispersión ocasionados por el aumento del entrehierro en las partes finales.

Las topologías dos y tres presentan ciertos comportamientos, como lo son perturbaciones en la 
forma de onda, desfases no deseados y una potencia de salida desbalanceada entre fases, que pueden ser tratados con componentes adicionales en la salida del generador pero que no hacen discutible la aplicabilidad de las mismas para bajas potencias.

Los patrones de magnetización tienen un papel importante en los generadores lineales dado que pueden mejorar el comportamiento de la tensión inducida en bornes del generador, y por ende, mejorar el rendimiento del mismo. Un arreglo bien configurado y dimensionado resulta en un generador con las mismas características.

Gracias a que el generador se define para bajas potencias los problemas que se presentan cuando se quiere generar grandes bloques de potencia, como, por ejemplo, un tamaño exagerado de la máquina para soportar los esfuerzos dada la baja velocidad de la parte móvil [5], desaparecen hasta el punto de poder ser despreciables e incluso de no presentarse.

\section{Agradecimientos}

Al Grupo de investigación en Compatibilidad Electromagnética GCEM de la Universidad Distrital Francisco José de Caldas.

\section{Referencias}

[1] L. Wei, T. Nakamura and K. Imai, "Development and Optimization of Low-Speed and HighEfficiency Permanent Magnet Generator for Micro Hydro-Electrical Generation System", ELSEVIER, Renewable Energy, Kyoto University, Japan, 2019. https://doi.org/10.1016/j.renene.2019.09.049

[2] M. Ardestani, N. Arish and H. Yaghobi, "A New HTS Dual Stator Linear Permanent Magnet Vernier Machine with Halbach Array for Wave Energy Conversion", ELSEVIER, Physyca C: Superconductivity and its Applications, Semman University, Iran, 2019. https://doi.org/10.1016/j.physc.2019.1353593
[3] P. Khatri and X. Wang, "Comprehensive Review of a Linear electrical Generator for Ocean Wave Energy Conversion", IET Renewable Power Generation, IET, Vol. 14, Lss. 6, pp. 949-958, February, 2020.

[4] O. S. Muñoz Muñoz, "Dimensionamiento electromagnético de un Generador Lineal para la Conversión de Energía Undimotriz de Acuerdo a las Características del Océano Pacífico Colombiano", trabajo de fin de grado, Universidad del Valle, Colombia, 2020.

[5] C. García Saiz, "Diseño, Dimensionado y Simulación de un Generador Lineal para el Desarrollo de una Boya de Generación de Energía Undimotriz", trabajo de fin de grado, Universidad de Cantabria, España, 2015. https://repositorio.unican.es/xmlui/handle/1090 $2 / 7332$

[6] A. García Villalmanzo, "Diseño de un Motor Lineal de Reluctancia Autoconmutado con Imanes Permanentes", trabajo de fin de grado, Universidad Rovira I Virgili, Tarragona, 2017. http://deeea.urv.cat/public/PROPOSTES/pub/pdf /2459pub.pdf

[7] A. Shiri and A. Shoulaie, "End Effect Braking Force Reduction in High-Speed Single-Sided Linear Induction Machine", ELSEVIER, Energy Conversion and Management, Iran University of Science and Technology, Iran, 2012. https://doi.org/10.1016/j.enconman.2011.11.01 4

[8] X. Chen, S. Zheng, J. Li, G. T. Ma and F. Yen, "A Linear Induction Motor with a Coated Conductor Superconducting Secondary", ELSEVIER, Physyca C: Superconductivity and its Applications, Southwest Jiaotong University, China, 2017. https://doi.org/10.1016/j.physc.2018.04.002

[9] SS. Rathore, S. Mishra, M. K. Paswan and Sanjay, "A Review on Design and Development of Free Piston Linear Generators in Hybrid Vehicles", 
IOP Conference Series: Materials Science and Engineering, ICCEMME, India, 2019.

[10] Y. Gao, S. Shao, H. Zou, M. Tang, H. Xu and C. Tian, "A Fully Floating System for Wave Energy Converter with Direct-Driven Linear Generator", ELSEVIER, Energy, Beijing, China, 2015. https://doi.org/10.1016/j.energy.2015.11.072

[11] J. F. Fortes, L. M. Ferraz and I. E. Chabu, "Optimized Double Sided Linear Generator for Wave Energy in Sao Paulo's Coast", 7th International Conference on Ocean Energy (ICOE), Polytechnic School of University of Sao Paulo, France, 2018. https://www.icoeconference.com/publication/optimized-doublesided-linear-generator-for-wave-energy-in-saopaulo-s-coast/

[12] V. Boscaino, G. Cipriani, V. Di Dio, V. Franzitta and M. Trapanense, "Experimental Test and Simulations on a Linear Generator-Based Prototype of a Wave Energy Conversion System Designed with a Reliability-Oriented Approach", MDPI, Sustainability, University or Palermo, 2017.

[13] O. Farrok, M. R. Islam, Y. G. Guo and J. G. Zhu, "Design and Analysis of a Novel Lightweight Translator Permanent Magnet Linear Generator for Oceanic Wave Energy Conversion", IEEE, 2015.

[14] K. Cruz, F. Dator, J. Ong, N. Bumanlag and M. C. Pacis, "Harnessing of Wave Energy using Axially Magnetized Linear Generator with Data Logger using Gizduino Microcontroller", IOP Conference Series: Journal of Physics: Conference Series, CEEPE, Mapua University, Philippines, 2019.

[15] A. Tapia-Hernández, M. Ponce-Silva, N. Mondragón-Escamilla y L. HernándezGonzález, "Impacto de la Geometría en el Efecto Fin de Generadores Lineales", Información Tecnológica, Vol.27, No. 4, pp. 133-138,
México, 2016. http://dx.doi.org/10.4067/S071807642016000400014

[16] P. Naderi, M. Heidary and M. Vahedi, "Performance Analysis of Ladder-SecondaryLinear Induction Motor with Two Different Secondary Types using Magnetic Equivalent Circuit", ELSEVIER, ISA Transactions, Shahid Beheshti University, Iran, 2020. https://doi.org/10.1016/j.isatra.2020.03.013

[17] Y. Xu, X. Xue, Y. Wang and M. Ai, "Performance Characteristics of Compressed Air-Driven-FreePiston Linear Generator (FPLG) System - A Simulation Study", ELSEVIER, Applied Thermal Engineering, 2019. https://doi.org/10.1016/j.applthermaleng.2019.1 14013

[18] J. G. Mora Santos, E. Rivas Trujillo and H. Montana Quintero, "Comparative Study of a Linear Permanent Magnet Generator of LowPower through the Finite Elements Method", International Journal of Mechanical and Production Engineering Research and Development, IJMPERD, Colombia, 2020.

[19] J. Xi, Z. Dong, P. Liu and H. Ding, "Modeling and Identification of Iron-less PMLSM End Effects for Reducing Ultra-Low-Velocity Fluctuations of Ultra-precision Air Bearing Linear Motion Stage", ELSEVIER, Precision Engineering, Shanghai Jiaotong University, China, 2017. https://doi.org/10.1016/j.precisioneng.2017.01. 016

[20] X. Luo, C. Zhang, S. Wang, E. Zio and X. Wang, "Modeling and Analysis of Mover Gaps in Tubular Moving-Magnet Linear Oscillating Motors", ELSEVIER, Chinese Journal of Aeronautics, Chinese Society of Aeronautics ans Astronautics \& Beihang University, China, 2017. https://doi.org/10.1016/j.cja.2017.11.008

[21] K. S. Rama Rao, T. Sunderan and M. Ref'at Adiris, "Performance and Design Optimization of Two Model Based Wave Energy Permanent Magnet 
Linear Generators", ELSEVIER, Renewable

Energy, 2016.

https://doi.org/10.1016/j.renene.2016.07.019

[22] M. F. M Naafi, T. Ibrahim, N. M. Nor and M. A. Firdaus bin M. Hamim, "Design and Modelling of a Portable Pico Linear Generator for Wave Energy Conversion System", Applied Mechanics and Materials, Vol. 785, pp. 300-304, Malaysia, 2015.

https://doi.org/10.4028/www.scientific.net/AM M.785.300

[23] W. Rentería Palacios, "Diseño y Evaluación Electromagnética de un Motor Síncrono Lineal de Imanes Permanentes en Disposición Halbach", trabajo de fin de máster, Universidad Autónoma de Occidente, Colombia, 2018. http://hdl.handle.net/10614/10454

[24] J. Kim, J. Y. Kim and J. B. Park, "Design and Optimization of a $8 \mathrm{~kW}$ Linear Generator for a Direct-Drive Point Absorber", IEEE, Yonsei University, Seoul, Korea, 2013.

[25] W. Li, T.W. Ching and K.T. Chau, "Design and Analysis of a New Parallel-Hybrid-Excited Linear
Vernier Machine for Oceanic Wave Power Generation", ELSEVIER, Applied Energy, China, 2017.

https://doi.org/10.1016/j.apenergy.2017.09.061

[26] L. Huang, J. Liu, H. Yu, R. Qu, H. Chen and H. Fang, "Winding Configuration and Performance Investigation of a Tubular Superconducting FluxSwitching Linear Generator", IEEE, Transactions on Applied Superconductivity, Vol. 25, No. 3, 2015.

[27] X. Liu, H. Yu, Z. Shi, T. Xia and M. Hu, "Electromagnetic-Fluid-Thermal Field Calculation and Analysis of a Permanent Magnet Linear Motor", ELSEVIER, Applied Thermal engineering, Southeast University, China, 2017. https://doi.org/10.1016/j.applthermaleng.2017.1 0.066

[28] X. Chen, S. Zheng, J. Li, G. T. Ma and F. Yen, "A Linear Induction Motor with a Coated Conductor Superconducting Secondary", ELSEVIER, Physyca C: Superconductivity and its Applications, Southwest Jiaotong University, China,

2017. 